\title{
Interference Graph-Based Resource-Sharing Schemes for Vehicular Networks
}

\author{
Rongqing Zhang, Student Member, IEEE, Xiang Cheng, Member, IEEE, Qi Yao, \\ Cheng-Xiang Wang, Senior Member, IEEE, Yang Yang, Senior Member, IEEE, and Bingli Jiao, Member, IEEE
}

\begin{abstract}
This paper investigates the resource-sharing problem in vehicular networks, including both vehicle-to-vehicle (V2V) and vehicle-to-infrastructure (V2I) communication links. A novel underlaying resource-sharing communication mode for vehicular networks is proposed, in which different $\mathrm{V} 2 \mathrm{~V}$ and V2I communication links are permitted to access the same resources for their individual data transmission. To solve the resource-sharing problem in vehicular networks, we, for the first time, apply graph theory and propose the following two interference graph-based resource-sharing schemes: 1) the interference-aware graph-based resource-sharing scheme and 2) the interferenceclassified graph-based resource-sharing scheme. Compared with the traditional orthogonal communication mode in vehicular networks, the proposed two resource-sharing schemes express better network sum rate. The utility of the proposed V2V and V2I underlaying communication mode and the two proposed interference graph-based resource-sharing schemes are verified by simulations.
\end{abstract}

Manuscript received June 15, 2012; revised October 16, 2012 and January 17, 2013; accepted January 17, 2013. Date of publication February 5, 2013; date of current version October 12, 2013. This work was supported in part by the National Natural Science Foundation of China under Grant 61101079 and Grant 61231009; the Ministry of Education of China through the Science Foundation for the Youth Scholar under Grant 20110001120129, through the Academic Scholarship for Doctoral Candidates, and through the Opening Project of Key Laboratory of Cognitive Radio and Information Processing (Guilin University of Electronic Technology) under Grant 2011KF01; the National Mobile Communications Research Laboratory through the Open Research Fund under Grant 2012D06, Southeast University; the Research Councils U.K. through the U.K.-China Science Bridges Project: R\&D on (B)4G Wireless Mobile Communications; the Shenzhen City Government through the Fundamental Research Program under Grant JCYJ20120817163755061; and the Science and Technology Commission of Shanghai Municipality under Grant 11JC1412300. The review of this paper was coordinated by Prof. M. Haenggi.

R. Zhang and X. Cheng are with the State Key Laboratory of Advanced Optical Communication Systems and Networks and the Wireless Communications and Signal Processing Research Center, School of Electronics Engineering and Computer Science, Peking University, Beijing 100871, China, and also with the National Mobile Communications Research Laboratory, Southeast University, Nanjing 210096, China (e-mail: rongqing.zhang@pku.edu.cn; xiangcheng@ pku.edu.cn).

Q. Yao and C.-X. Wang are with the School of Information Science and Engineering, Shandong University, Shandong 250100, China, and the Joint Research Institute for Signal and Image Processing, School of Engineering and Physical Sciences, Heriot-Watt University, Edinburgh EH14 1AS, U.K. (e-mail: q.yao@hw.ac.uk; cheng-xiang.wang@hw.ac.uk).

Y. Yang is with the Shanghai Institute of Microsystem and Information Technology, the Shanghai Research Center for Wireless Communications, and also with the Chinese Academy of Sciences (CAS) Key Laboratory of Wireless Sensor Network and Communication, ShanghaiTech University, Shanghai 200031, China (e-mail: yang.yang@ shrcwc.org).

B. Jiao is with the State Key Laboratory of Advanced Optical Communication Systems and Networks and the Wireless Communications and Signal Processing Research Center, School of Electronics Engineering and Computer Science, Peking University, Beijing 100871, China (e-mail: jiaobl@pku. edu.cn).

Color versions of one or more of the figures in this paper are available online at http://ieeexplore.iee.org.

Digital Object Identifier 10.1109/TVT.2013.2245156
Index Terms-Interference-aware graph, interference-classified graph, resource sharing, vehicle-to-infrastructure (V2I) communication, vehicle-to- vehicle (V2V) communication.

\section{INTRODUCTION}

$\mathbf{R}$ ECENTLY, vehicular networks have attracted considerable attention due to the emergence of intelligent transportation systems (ITSs). To support various ITS applications, both vehicle-to-infrastructure (V2I) and vehicle-to-vehicle (V2V) communications need to be supported in vehicular networks [1]-[7]. On one hand, V2I communications allow the vehicles to access the roadside infrastructure (RSU) that belongs to one or several service providers for downloading or uploading various types of data related to different applications. On the other hand, V2V communications enable a pair or group of vehicles to communicate and exchange information for different purposes. However, most previous works on the investigation and analysis of vehicular networks have mainly focused on either V2V or V2I communication scenarios [8]-[10]. For example, in [8], scheduling with quality-of-service (QoS) support for V2I communications is focused upon, and an optimal control-based algorithm is proposed that relies on the polling-based contention-free access mode of the IEEE 802.11e Standard. In [9], a joint power and subcarrier assignment policy under delay-aware QoS requirements, which can effectively improve the power efficiency in V2I communication networks, is proposed. In [10], a low-complexity outage-optimal distributed channel allocation for $\mathrm{V} 2 \mathrm{~V}$ communications by solving a maximum matching problem based on a random bipartite graph, is proposed.

More recently, to achieve a further-improved network performance in vehicular networks, many more researchers have been starting to concentrate on the design of vehicular networks through the joint consideration of both V2V and V2I communications [11]-[15]. Based on [11]-[15], it has been demonstrated that, compared with vehicular networks using either V2V or V2I communications, the performance of vehicular networks can further be improved by jointly considering both V2V and V2I communications. In [11], the information spread problem in a joint V2I and V2V communication system is investigated by using network coding at the infrastructure and the vehicles to improve the system throughput. In [12], the access probability and connectivity probability performance in a vehicular relay network, considering both one-hop (V2I) and two-hop (V2I and V2V) communications between a vehicle and the infrastructure is studied. In [13], for the first time, a 
vehicle-to-vehicle-to-infrastructure (V2V2I) architecture for vehicular networks, which is a hybrid of V2I and V2V architectures, is described. A super-vehicle algorithm is then proposed in [14] for the V2V2I communication system, where the vehicular network is broken into several preconfigured zones, and in each zone, there exists a super vehicle. The super vehicle can receive data from all of the other vehicles within its zone, aggregate the data, and then transmit the aggregated data to the central server or other super vehicles in adjacent zones. In [15], the V2V2I communication system in vehicular networks is improved by utilizing smart-antenna-based beamforming and proposed a downlink resource allocation scheme to mitigate the cochannel interference between V2I and V2V communications.

To the best of the authors' knowledge, there is no work available in public to deal with the resource-sharing issue in vehicular networks from a media access and control (MAC)layer perspective, i.e., different V2V and/or V2I communications can share the same radio resources for their individual data transmission with the cochannel interference limited by proper resource assignment among different communication links. The utility of a network with such a resource-sharing mechanism has been proved in [16] and [17], where device-to-device (D2D) communication is used as an underlay to cellular networks and, thus, can share the same resources with the traditional cellular communication. This D2D communication underlaying cellular network has proved to be an efficient communication mode that can alleviate the communication load of the base station (BS) and thus further improve the network efficiency [16], [17].

Motivated by this case, in this paper, we investigate the resource-sharing problem from a MAC-layer perspective in vehicular networks where V2V and V2I communications coexist and act as an underlay to each other. In particular, we first propose a novel underlaying resource-sharing communication mode for vehicular networks in which different V2V and V2I communication links are permitted to access the same resources for their individual data transmission. Then, we formulate the resource-sharing problem as a resource assignment optimization problem, considering the interference between different communication links. However, optimally solving the resource assignment optimization problem is computationally prohibitive. Graph theory has been widely accepted as a useful tool for modeling and analyzing various types of interactions, relations, and dynamics in different networks [18] and has been widely used in the design and analysis of resource management problems in cellular networks [19]-[21]. Therefore, by using the graph theory, we propose the following two interference graph-based resource-sharing schemes to effectively obtain a suboptimal resource assignment solution in low computational complexity: 1) the interference-aware graph-based resource-sharing scheme and 2) the interference-classified graph-based resource-sharing scheme. The simulation results show that, compared with the traditional orthogonal communication mode in vehicular networks, the proposed resourcesharing schemes have significantly better network sum rate, which indicates the efficiency in improving the network performance by employing the underlaying resource-sharing communication mode into the vehicular networks. The per- formance comparison also verifies that the proposed interference graph-based resource-sharing schemes can achieve proper and effective resource assignment among different communication links and thus help in efficiently realizing the performance advantage of the underlaying resource-sharing communication mode in vehicular networks. The novelties and main contributions of this paper can be summarized as follows.

1) From a resource management perspective in the MAC layer, we propose an underlaying resource-sharing communication mode for vehicular networks where both $\mathrm{V} 2 \mathrm{~V}$ and V2I communications coexist and are permitted to access the same resources for their individual data transmission. The proposed underlaying resource-sharing communication mode can effectively improve the spectrum efficiency of vehicular networks.

2) To deal with the optimal resource-sharing problem in low computational complexity, the graph theory is, for the first time, applied to design and analyze resource-sharing problems in vehicular networks. To achieve near-optimal performance, we propose an interference-aware graphbased resource-sharing scheme.

3) To further reduce the high communication overhead of the proposed interference-aware graph-based resource-sharing scheme, we propose a resource-sharing scheme that is simpler and easier to implement, i.e., an interference-classified graph-based resource-sharing scheme. The proposed interference-classified graphbased resource-sharing scheme can also achieve suboptimal performance and shows better network sum rate over the traditional orthogonal communication mode.

The rest of this paper is organized as follows. In Section II, the underlaying resource-sharing communication mode for vehicular networks is proposed, and the resource-sharing problem is formulated as a resource assignment optimization problem. In Section III, the interference-aware graph-based resourcesharing scheme is proposed. In Section IV, the interferenceclassified graph-based resource-sharing scheme is proposed. Simulation results are provided in Section V, and the conclusions are drawn in Section VI.

\section{System Model and Problem Formulation}

\section{A. System Description}

Consider a vehicular network where both V2I and V2V communication links coexist, as illustrated in Fig. 1. The investigated vehicular network consists of one RSU that can deliver information to the vehicles within its broadcasting coverage, $M$ vehicles that access the RSU for the information that they demand, and $N$ pairs of vehicles that communicate with each other. Therefore, there exist $M$ V2I and $N$ V2V communication links in the network, denoted by $A_{m}$ and $B_{n}$, respectively, where $m=1,2, \ldots, M$, and $n=1,2, \ldots, N$. Denote the set of indices $\{1,2, \ldots, M\}$, the set of indices $\{1,2, \ldots, N\}$, the set of V2I communication links, and the set of V2V communication links by $\mathcal{M}, \mathcal{N}, \mathcal{A}$, and $\mathcal{B}$, respectively. Each vehicle and 


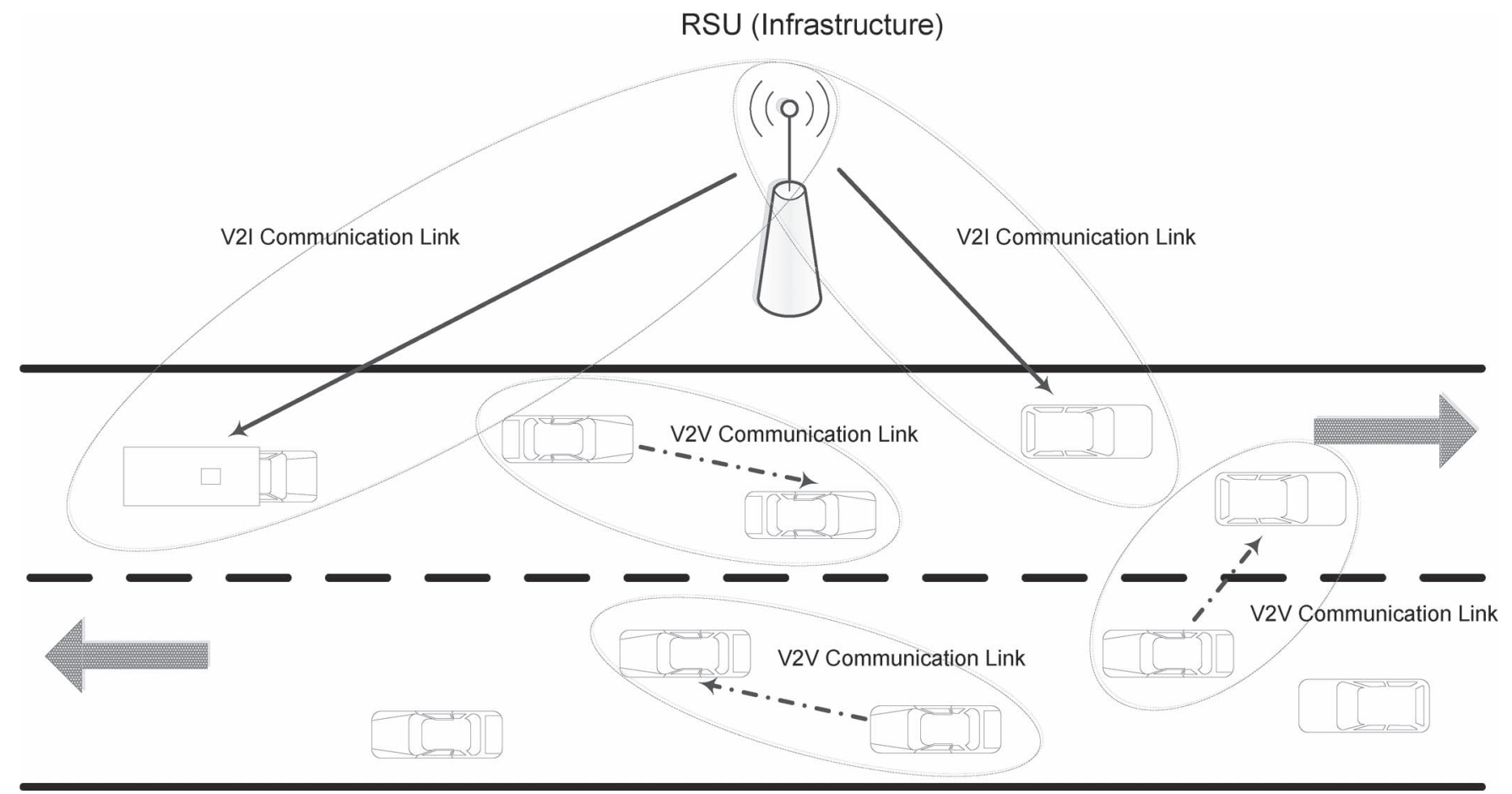

Fig. 1. Vehicular network where both V2I and V2V communication links coexist.

the RSU are equipped with a single omnidirectional antenna and operate in a half-duplex manner. At the physical layer, the orthogonal frequency-division multiplexing (OFDM) technique is employed to support multiple accesses for both V2I and V2V data transmission. Then, the total channel bandwidth $W$ can be divided into $K$ orthogonal resource blocks (RBs), denoted by $\mathcal{R}=\left\{\mathrm{RB}_{1}, \mathrm{RB}_{2}, \ldots, \mathrm{RB}_{K}\right\}$, where each $\mathrm{RB}$ occupies a certain number of orthogonal subcarriers.

To improve the spectrum efficiency of the considered vehicular network, which consists of both V2I and V2V communication links, we propose an underlaying resource-sharing communication mode in which different V2I and V2V communication links are permitted to access the same resources, i.e., RBs, for their individual data transmission. The proposed underlaying communication mode can offer the possibility of effectively increasing the throughput of the vehicular network through efficient interference management protocols. Note that, although the proposed underlaying communication mode is similar to the concept of D2D communications used as an underlay to cellular networks, it is different from the D2D communication underlaying cellular network in the priority issue [16], i.e., in the proposed underlaying communication mode, V2I and V2V communication links have the same priority to obtain RBs for data transmission, which means that $\mathrm{V} 2 \mathrm{I}$ and $\mathrm{V} 2 \mathrm{~V}$ communications perform as an underlay to each other, whereas in a D2D communication underlaying cellular network, traditional cellular communication links have higher priority than D2D communication links when accessing the radio resources. Because this paper investigates the downlink transmission case for V2I communications, i.e., the RSU transmits information to the corresponding vehicles, different V2I communication links should not be allowed to share the same RB for their individual data transmission, because all the V2I communication links have the same transmitter, i.e., the RSU. If different V2I communication links can share the same RB, the information for different vehicles would be mixed up at the transmitter, making it difficult for each vehicle to distinguish its demanded information from the superimposed data symbols. Therefore, resource sharing among different V2I communication links is forbidden in the proposed underlaying communication mode.

The key issue of the use of the proposed underlaying resource-sharing communication mode is the proper design of interference management protocol. An efficient interference management protocol for such an underlaying resource-sharing scenario can be achieved by properly clustering V2I and V2V communication links for different RBs, considering interference among them. The RSU will act as a scheduling operator that can collect the communication requests from individual vehicles that require a V2I downlink data transmission link and pairs of the vehicles that expect to set up a $\mathrm{V} 2 \mathrm{~V}$ communication link, allocate the RBs to different communication links based on efficient resource-sharing algorithms, and announce the decisions on RB allocation to the vehicles within its communication coverage. Once the RB allocation has been decided, the data transmission of each communication link can be performed over the allocated RBs. Note that, for simplicity and with respect to fairness for different communication links to acquire communication service, we assume that each communication link can be allocated at most one RB in an OFDM symbol for its data transmission through every scheduling process. As illustrated in [17] and [20], the aforementioned assumption has been widely used on the studies of resource-sharing issues in OFDM systems. 


\section{B. Resource-Sharing Problem Formulation}

Suppose that the RSU and the vehicles transmit with power $p_{r}$ and $p_{v}$, respectively, and the transmit power of the RSU allocated to each RB for V2I data transmission is equal, i.e., the transmit power on each $\mathrm{RB}$ is $p_{r} / K$. The channel gains of the V2I $A_{m}$ and $\mathrm{V} 2 \mathrm{~V} B_{n}$ communication links on $\mathrm{RB}_{k}$ are denoted by $g_{A_{m}}^{k}$ and $g_{B_{n}}^{k}$, respectively, where $m \in \mathcal{M}, n \in \mathcal{N}$, and $k \in \mathcal{K}$. The channel gains of the interference links from the V2I communication link $A_{m}$ to the V2V communication link $B_{n}$, from the $\mathrm{V} 2 \mathrm{~V}$ communication link $B_{n}$ to the V2I communication link $A_{m}$, and from one $\mathrm{V} 2 \mathrm{~V}$ communication link $B_{n}$ to another $\mathrm{V} 2 \mathrm{~V}$ communication link $B_{n^{\prime}}$ when they share $\mathrm{RB}_{k}$ for individual data transmission are represented by $g_{A_{m}, B_{n}}^{k}, g_{B_{n}, A_{m}}^{k}$, and $g_{B_{n}, B_{n^{\prime}}}^{k}$, respectively, where $m \in$ $\mathcal{M}, n \in \mathcal{N}, n^{\prime} \in \mathcal{N}, n \neq n^{\prime}$, and $k \in \mathcal{K}$. In this paper, the channel gains contain the distance-dependent path loss and the normalized small-scale fading, i.e., $g_{A_{m}}^{k}=P L_{A_{m}} h_{A_{m}}^{k}$, $g_{B_{n}}^{k}=P L_{B_{n}} h_{B_{n}}^{k}, \quad g_{A_{m}, B_{n}}^{k}=P L_{A_{m}, B_{n}} h_{A_{m}, B_{n}}^{k}, \quad g_{B_{n}, A_{m}}^{k}=$ $P L_{B_{n}, A_{m}} h_{B_{n}, A_{m}}^{k}$, and $g_{B_{n}, B_{n^{\prime}}}^{k}=P L_{B_{n}, B_{n^{\prime}}} h_{B_{n}, B_{n^{\prime}}}^{k}$, where $P L_{A_{m}}, P L_{B_{n}}, P L_{A_{m}, B_{n}}, P L_{B_{n}, A_{m}}$, and $P L_{B_{n}, B_{n^{\prime}}}$ represent the corresponding distance-dependent path loss, and $h_{A_{m}}^{k}, h_{B_{n}}^{k}$, $h_{A_{m}, B_{n}}^{k}, h_{B_{n}, A_{m}}^{k}$, and $h_{B_{n}, B_{n^{\prime}}}^{k}$ represent the corresponding normalized small-scale channel fading on the $\mathrm{RB}_{k}$. Note that, in our assumption, the small-scale fading on different $\mathrm{RBs}$ for a certain communication link is independent, but within one RB, it stays the same, i.e., a flat fading in each RB [9]. The thermal noise at the vehicles satisfies independent Gaussian distribution with zero mean and the same variance and is denoted by $\sigma^{2}$.

The instantaneous signal-to-interference-plus-noise ratio (SINR) at the receiver of the V2I communication link $A_{m}$, $m \in \mathcal{M}$ when the $\mathrm{RB}_{k}$ is allocated to it for data transmission can be given as

$$
\operatorname{SINR}_{A_{m}}^{k}=\frac{\frac{p_{r}}{K} g_{A_{m}}^{k}}{\sigma^{2}+\sum_{j \in \mathcal{N}, B_{j} \in \mathcal{C}_{k}} p_{v} g_{B_{j}, A_{m}}^{k}}
$$

and the instantaneous SINR at the receiver of the V2V communication link $B_{n}, n \in \mathcal{N}$ when the $\mathrm{RB}_{k}$ is allocated to it for data transmission can be given as

$$
\begin{aligned}
\operatorname{SINR}_{B_{n}}^{k} & =\frac{p_{v} g_{B_{n}}^{k}}{\sigma^{2}+\sum_{I \in \mathcal{M}, A_{i} \in \mathcal{C}_{k}} \frac{p_{r} g_{A_{i}, B_{n}}^{k}}{K}+\sum_{j \in \mathcal{N}, j \neq n, B_{j} \in \mathcal{C}_{k}} p_{v} g_{B_{j}, B_{n}}^{k}}
\end{aligned}
$$

where $\mathcal{C}_{k}$ represents the cluster of the communication links that share the $\mathrm{RB}_{k}$ for individual data transmission.

The key issue of the resource-sharing problem is to find the optimal RB assignment solution for V2I and V2V communication links, based on which the communication links can properly perform their individual data transmission in the underlaying vehicular network. By applying the Shannon capacity formula, we define the sum rate of the vehicular network as the sum of the channel capacity for all V2I and V2V communication links within the network. Let $\mathbf{S}_{(M+N) \times K}=\left(\begin{array}{c}\mathbf{X}_{M \times K} \\ \mathbf{Y}_{N \times K}\end{array}\right)$ be an RB assignment solution, where $\mathbf{X}_{M \times K}=\left[\alpha_{m, k}\right]$ and
$\mathbf{Y}_{N \times K}=\left[\beta_{n, k}\right]$ denote the RB assignment matrix for V2I and V2V communication links, respectively. The value of $\alpha_{m, k}$ and $\beta_{n, k}$, with $m \in \mathcal{M}, n \in \mathcal{N}$, and $k \in \mathcal{K}$, can be defined as

$$
\begin{aligned}
\alpha_{m, k} & = \begin{cases}1, & \text { when } \mathrm{RB}_{k} \text { allocated to } A_{m} \\
0, & \text { otherwise }\end{cases} \\
\beta_{n, k} & = \begin{cases}1, & \text { when } \mathrm{RB}_{k} \text { allocated to } B_{n} \\
0, & \text { otherwise. }\end{cases}
\end{aligned}
$$

Therefore, we can obtain the optimal RB assignment solution, denoted by $\mathbf{S}_{o p t}$, by solving the optimization problem as

$$
\begin{aligned}
& \mathbf{S}_{\text {opt }}= \arg \max _{\mathbf{S}_{(M+N) \times K}} \sum_{k=1}^{K} \frac{W}{K} \\
& \times\left[\sum_{m=1}^{M} \log _{2}\left(1+\mathrm{SINR}_{A_{m}}^{k}\right) \alpha_{m, k}\right. \\
&\left.+\sum_{n=1}^{N} \log _{2}\left(1+\mathrm{SINR}_{B_{n}}^{k}\right) \beta_{n, k}\right] \\
& \text { s.t. } \quad\left\{\begin{array}{l}
\mathcal{C}_{k}=\left\{A_{i}, B_{j} \mid \alpha_{i, k}=1, \beta_{j, k}=1, i \in \mathcal{M}, j \in \mathcal{N}\right\} \\
\sum_{m=1}^{M} \alpha_{m, k} \leq 1 \\
\sum_{k=1}^{K} \alpha_{m, k} \leq 1 \quad \sum_{k=1}^{K} \beta_{n, k} \leq 1
\end{array}\right.
\end{aligned}
$$

where $\operatorname{SINR}_{A_{m}}^{k}$ and $\operatorname{SINR}_{B_{n}}^{k}$ are given in (1) and (2), respectively. Note that the second constraint in (6) guarantees that the situation that two or more V2I communication links occupy the same RB for data transmission is forbidden and the third constraint in (6) guarantees that each communication link can obtain at most one RB in an OFDM symbol through every RB assignment process. In addition, note that, in this paper, we mainly focus on the resource assignment problem in the underlaying vehicular network to optimize the network sum rate at a certain scheduling time point. Thus, the fairness problem among different communication links is not within our consideration, which can effectively be solved by employing the well-known proportional fair (PF) protocol [22], [23] to design a PF scheduler along the time dimension based on the optimization criterion that we formulated in (5).

The optimal RB assignment solution in (5) can be obtained through an exhaustive search for all the possible choices of $\alpha_{m, k}$ and $\beta_{n, k}$ subject to the conditions given in (6), where $\alpha_{m, k}$ and $\beta_{n, k}$ are independent of each other. Then, the computational complexity can be obtained as the multiplication of the complexity with all possible choices of $\alpha_{m, k}$ and the complexity with all possible choices of $\beta_{n, k}, m \in \mathcal{M}, n \in \mathcal{N}$, and $k \in \mathcal{K}$.

After further calculations, the computational complexity of the optimization problem formulated in (5) and (6) can be obtained as

$$
\mathcal{C}_{\text {optimal }}=\mathcal{O}\left(\sum_{k=0}^{K} \mathcal{P}_{M}^{k} \cdot \frac{(N+1)^{K-1}}{(K-1) !}\right)
$$


where $(x)$ ! denotes the factorial computation of a nonnegative integer $x$, and $\mathcal{P}_{x}^{y}$ represents the permutation operation, which is defined as

$$
\mathcal{P}_{x}^{y}= \begin{cases}\frac{(x) !}{(x-y) !}, & \text { when } x \geq y \\ 0, & \text { otherwise }\end{cases}
$$

where $x$ and $y$ are both nonnegative integers, and we further define $(0) !=1$.

Based on (7), the optimization problem that was formulated in (5) and (6) is an NP-hard combinatorial optimization problem with nonlinear constraints [24]; in particular, as the number of V2I and V2V communication links (i.e., $M$ and $N$ ) grows, the computational complexity rapidly increases. In other words, directly finding an optimal solution of the aforementioned optimization problem is computationally prohibitive, and thus, no polynomial-time algorithm can optimally solve it. Therefore, in the following sections, we propose two interference graphbased resource-sharing schemes to effectively obtain a suboptimal RB assignment solution in low computational complexity to achieve an improved sum rate of the V2I and V2V underlaying communication mode compared with the traditional orthogonal communication mode in vehicular networks.

\section{INTERFERENCE-AWARE GRAPH-BASED RESOURCE-SHARING SCHEME}

In this section, we propose an interference-aware graphbased resource-sharing scheme for the V2I and V2V communication underlaying vehicular network. The interference-aware case is defined as a condition that the RSU can acquire local awareness on the channel gains of each communication link and between different communication links, making it able to take this information into account when assigning the radio resources to $\mathrm{V} 2 \mathrm{I}$ and $\mathrm{V} 2 \mathrm{~V}$ communication links. The interference-aware condition can be achieved by applying the following approach: The RSU and each vehicle as a transmitter in V2I and V2V communication links would periodically broadcast sounding signals. The vehicles as receivers in communication links could receive the sounding signals from the transmitters if they are within the transmitters' communication coverage; then, they would estimate the channel gains of their own communication links and the interference links and report the channel information that they acquired to the RSU. If a receiver could not receive the sounding signal from a certain transmitter, which means it is out of the communication coverage of the transmitter, it would report the interference channel gain as 0 to the RSU. The RSU collects all the channel information reported by the vehicles, and hence, it could become an interference-aware scheduling operator.

\section{A. Interference-Aware Graph Construction}

The first step of the interference graph-based scheme for resource sharing in the V2I and V2V underlaying vehicular network is to construct the interference graph that corresponds to the network topology. Considering an illustrative example with two V2I communication links and three V2V communication links, as shown in Fig. 1, we construct a corresponding

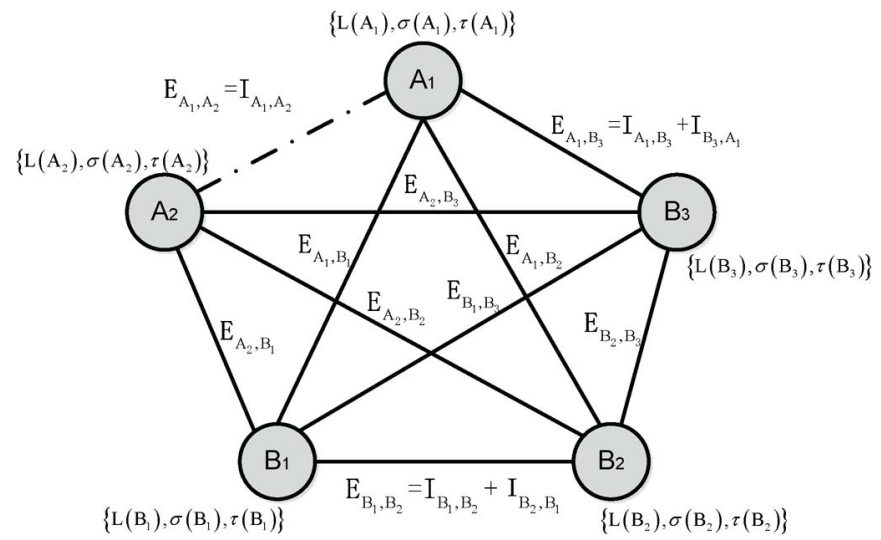

Fig. 2. Illustrative example of an interference-aware graph.

interference-aware graph as shown in Fig. 2, where the two V2I and three $\mathrm{V} 2 \mathrm{~V}$ communication links are denoted by $A_{1}, A_{2}$, $B_{1}, B_{2}$, and $B_{3}$, respectively. In the interference-aware graph, denoted by $\mathcal{G}_{i a}=\left(\mathcal{V}, \mathcal{E}_{i a}\right)$, each vertex in the vertices set $\mathcal{V}$ represents a V2I or V2V communication link, and each edge, which connects two vertices, in the edge set $\mathcal{E}_{i a}$ has a weight that characterizes the potential mutual interference between the two vertices.

Each vertex in the proposed interference-aware graph, denoted by $V_{i}$, with $i=1,2, \ldots, M+N$, has the following three individual attributes: 1) the link attribute, 2) the resource attribute, and 3) the cluster attribute. The link attribute indicates that the vertex belongs to either a V2I or a V2V communication link, i.e., $V_{i} \in \mathcal{A}$ or $V_{i} \in \mathcal{B}$, which affects the weight value calculation of the edges between different vertices. The resource attribute contains the following two parameters: 1) the $\mathrm{RB}$ index list $\mathcal{L}\left(V_{i}\right)$ and 2) the current interested RB index $\delta\left(V_{i}\right)$. The term $\mathcal{L}\left(V_{i}\right)$ is a vector of $\mathrm{RB}$ indices, ordered such that the RB index that corresponds to the highest signal-tonoise ratio (SNR) at the receiver appears first and the RB index that corresponds to the lowest SNR appears last, and $\delta\left(V_{i}\right)$ is the first element in $\mathcal{L}\left(V_{i}\right)$. The cluster attribute, denoted by $\tau\left(V_{i}\right)$, represents the cluster of RB to which the vertex currently belongs, i.e., if $V_{i} \in \mathcal{C}_{k}, k \in \mathcal{K}$, then $\tau\left(V_{i}\right)=k$. Note that, if $V_{i}$ does not currently belong to any cluster of $\mathrm{RB}$, then $\tau\left(V_{i}\right)=0$.

Different from many other interference graphs that were constructed for resource allocation problems [19]-[21], the edge weight $\mathbf{E}_{V_{i}, V_{j}}$ with $i \neq j$, which represents the potential mutual interference between two vertices $V_{i}$ and $V_{j}$, in the proposed interference-aware graph is a $K \times K$ interference matrix rather than a certain value, as shown in [19]-[21]. This is because we apply the more real assumption that channel responses on different $\mathrm{RBs}$ for a communication or interference link are different. We define the value of the edge weight as the sum of the mutual interference between two vertices, i.e., $\mathbf{E}_{V_{i}, V_{j}}=\mathbf{I}_{V_{i}, V_{j}}+\mathbf{I}_{V_{j}, V_{i}}$, where $\mathbf{I}_{V_{i}, V_{j}}$ and $\mathbf{I}_{V_{j}, V_{i}}$ represent the interference matrices from $V_{i}$ to $V_{j}$ and from $V_{j}$ to $V_{i}$, with $V_{i}, V_{j} \in \mathcal{V}$ and $i \neq j$, respectively. Note that, if both $V_{i}$ and $V_{j}$ are the V2I communication links, then the interference between them should be set to infinity or equal to a sufficiently large value to guarantee our assumption that resource sharing among 
different V2I communication links is forbidden. In addition, note that, if and only if two different communication links occupy the same RB, they can generate interference to each other. Otherwise, the interference between them will be zero. To determine the edge weight of the proposed interference-aware graph, we first need to obtain the interference matrices, which is given as

$$
\mathbf{I}_{V_{i}, V_{j}}=\left(\begin{array}{cccc}
I_{V_{i}, V_{j}}^{1} & 0 & \cdots & 0 \\
0 & I_{V_{i}, V_{j}}^{2} & \cdots & 0 \\
\vdots & \vdots & \ddots & \vdots \\
0 & 0 & \cdots & I_{V_{i}, V_{j}}^{K}
\end{array}\right)
$$

where $I_{V_{i}, V_{j}}^{k}$ represents the interference between $V_{i}$ and $V_{j}$ when they share $\mathrm{RB}_{k}$ with $k \in \mathcal{K}$ for data transmission. Based on the interference channel information collected by the RSU in our interference-aware assumption, $I_{V_{i}, V_{j}}^{k}$ can accurately be calculated as

$$
I_{V_{i}, V_{j}}^{k}= \begin{cases}\infty, & V_{i} \in \mathcal{A}, V_{j} \in \mathcal{A} \\ \frac{p_{r}}{K} g_{V_{i}, V_{j}}^{k}, & V_{i} \in \mathcal{A}, V_{j} \in \mathcal{B} \\ p_{v} g_{V_{i}, V_{j}}^{k}, & V_{i} \in \mathcal{B} .\end{cases}
$$

Then, the value of the edge weight $\mathbf{E}_{V_{i}, V_{j}}$ in the proposed interference graph can be obtained as

$$
\begin{aligned}
& \mathbf{E}_{V_{i}, V_{j}}
\end{aligned}
$$

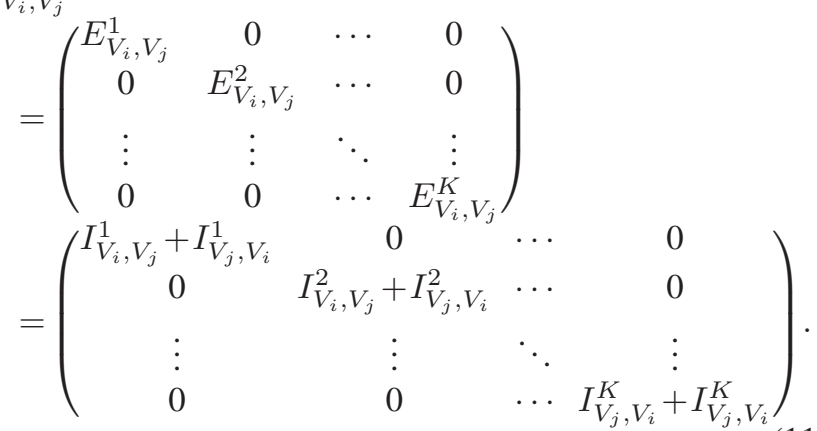

\section{B. Interference-Aware Graph-Based Resource-Sharing Algorithm}

Based on the interference-aware graph constructed in Section III-A, in this section, we propose an interference-aware graph-based resource-sharing algorithm to effectively assign RBs to different V2I and V2V communication links to improve the sum rate of the vehicular network.

To process the proposed interference-aware graph-based algorithm, we require several definitions as follows.

Definition 1: The cluster value of $\mathrm{RB}_{k}$ 's cluster $\mathcal{C}_{k}$, which is denoted by $v_{c}\left(\mathcal{C}_{k}\right)$, is defined as the sum of the channel capacity for all the communication links that belong to the cluster, taking the mutual interference among them into account. Therefore, the cluster value $v_{c}\left(\mathcal{C}_{k}\right)$ can be given as

$$
v_{c}\left(\mathcal{C}_{k}\right)=\sum_{V_{I} \in \mathcal{C}_{k}} \frac{W}{K} \log _{2}\left(1+\operatorname{SINR}_{V_{i}}^{k}\right)
$$

TABLE I

ALGORITHM 1: INTERFERENCE-AWARE GRAPH-BASED RESOURCE-SHARING ALGORITHM

1. Construct the interference-aware graph and initialize the parameters of the interference-aware graph.

$\star$ Calculate each edge weight $\mathbf{E}_{V_{i}, V_{j}}$ with $V_{i}, V_{j} \in \mathcal{V}$ and $V_{i} \neq$ $V_{j}$

* Initialize each vertex's individual information, i.e., $\mathcal{L}\left(V_{i}\right)$ and $\delta\left(V_{i}\right)$, and set $\tau\left(V_{i}\right)=0$ with $V_{i} \in \mathcal{V}$;

$\star$ Set $\mathcal{C}_{k}=\Phi$, where $\Phi$ represents an empty set, and $v_{i}\left(\mathcal{C}_{k}\right)=$ $v_{c}\left(\mathcal{C}_{k}\right)=0$ with $k \in \mathcal{K}$;

$\star$ Initialize $\mathcal{C}_{k}^{\star}=\left\{V_{i} \mid \delta\left(V_{i}\right)=k, V_{i} \in \mathcal{V}\right\}$ with $k \in \mathcal{K}$.

2. Repeat

$\star$ Select a vertex $V^{*}$ from the virtual cluster $\mathcal{C}_{k}^{\star}$ with $k \in \mathcal{K}$ :

- If $\mathcal{C}_{k}=\Phi$, then $V^{*}=\arg \max _{V_{i} \in \mathcal{C}^{\star}} v_{c}\left(\mathcal{C}_{k} \bigcup\left\{V_{i}\right\}\right)$;

- Else, $V^{*}=\arg \min _{V_{i} \in \mathcal{C}_{k}^{\star}} v_{i}\left(\mathcal{C}_{k} \bigcup\left\{V_{i}\right\}\right)$

* Compare the cluster value $v_{c}\left(\mathcal{C}_{k}\right)$ with $v_{c}\left(\mathcal{C}_{k} \bigcup\left\{V^{*}\right\}\right)$ :

- If $v_{c}\left(\mathcal{C}_{k} \bigcup\left\{V^{*}\right\}\right)>v_{c}\left(\mathcal{C}_{k}\right)$, then $\mathcal{C}_{k}=\mathcal{C}_{k} \bigcup\left\{V^{*}\right\}$, $\tau\left(V^{*}\right)=k$, and $\delta\left(V^{*}\right)=0$;

- Else, delete the first index in $\mathcal{L}\left(V^{*}\right)$ and update $\delta\left(V^{*}\right)$.

$\star$ Update all the virtual clusters.

Until $\mathcal{C}_{1}^{\star} \cup \mathcal{C}_{2}^{\star} \cup \cdots \cup \mathcal{C}_{K}^{\star}=\Phi$.

3. The RB assignment solution is composed of $\left\{\tau\left(V_{i}\right) \mid V_{i} \in \mathcal{V}\right\}$.

where $\operatorname{SINR}_{V_{i}}^{k}$ can be calculated by (1) or (2), depending on $V_{i} \in \mathcal{A}$ or $V_{i} \in \mathcal{B}$.

Definition 2: The interference value of $\mathrm{RB}_{k}$ 's cluster $\mathcal{C}_{k}$, denoted by $v_{i}\left(\mathcal{C}_{k}\right)$, is defined as the sum of the mutual interference between every two communication links that belong to the cluster. Therefore, the interference value $v_{i}\left(\mathcal{C}_{k}\right)$ can be given as

$$
\begin{aligned}
v_{i}\left(\mathcal{C}_{k}\right) & =\sum_{V_{i}, V_{j} \in \mathcal{C}_{k}, V_{i} \neq V_{j}}\left(I_{V_{i}, V_{j}}^{k}+I_{V_{j}, V_{i}}^{k}\right) \\
& =\sum_{V_{i}, V_{j} \in \mathcal{C}_{k}, V_{i} \neq V_{j}} E_{V_{i}, V_{j}}^{k}
\end{aligned}
$$

where $E_{V_{i}, V_{j}}^{k}$ can easily be obtained from the edge weight of the proposed interference graph.

Definition 3: The virtual cluster of $\mathrm{RB}_{k}$, denoted by $\mathcal{C}_{k}^{\star}$, is defined as the set of the vertices whose current interested $\mathrm{RB}$ index is $k$, i.e., $\mathcal{C}_{k}^{\star}=\left\{V_{i} \mid \delta\left(V_{i}\right)=k, V_{i} \in \mathcal{V}\right\}$.

The basic idea of the proposed interference-aware graphbased resource-sharing algorithm is to iteratively gather vertices from the virtual clusters into the corresponding clusters of the same RB, taking both the interference and cluster values into account to guarantee that the cluster value of each cluster is maximized.

As described in Algorithm 1, which is shown in Table I, at the start of the resource assignment process, the interferenceaware graph that indicates the current situation of the vehicular network is constructed based on the channel and identity information collected from the V2I and V2V communication links by the RSU. The parameters of the interference-aware graph are initialized as follows: Calculate each edge weight $\mathbf{E}_{V_{i}, V_{j}}$ with $V_{i}, V_{j} \in \mathcal{V}$, and $V_{i} \neq V_{j}$. Initialize each vertex's individual information, i.e., $\mathcal{L}\left(V_{i}\right)$ and $\delta\left(V_{i}\right)$, and set $\tau\left(V_{i}\right)=0$ with $V_{i} \in \mathcal{V}$. Set $\mathcal{C}_{k}=\Phi$, where $\Phi$ represents an empty set, and $v_{i}\left(\mathcal{C}_{k}\right)=v_{c}\left(\mathcal{C}_{k}\right)=0$, with $k \in \mathcal{K}$. Initialize $\mathcal{C}_{k}^{\star}=\left\{V_{i} \mid \delta\left(V_{i}\right)=\right.$ $\left.k, V_{i} \in \mathcal{V}\right\}$, with $k \in \mathcal{K}$. 
Considering the cluster $\mathcal{C}_{k}$ of $\mathrm{RB}_{k}$, we first select a vertex $V^{*}$ from the virtual cluster $\mathcal{C}_{k}^{\star}$ to satisfy $V^{*}=$ $\arg \min _{V_{i} \in \mathcal{C}_{k}^{\star}} v_{i}\left(\mathcal{C}_{k} \cup\left\{V_{i}\right\}\right)$. Note that, if $\mathcal{C}_{k}=\Phi$, the selected vertex should be the vertex that can achieve the largest cluster value in $\mathcal{C}_{k}$, i.e., $V^{*}=\arg \max _{V_{i} \in \mathcal{C}_{k}^{\star}} v_{c}\left(\mathcal{C}_{k} \cup\left\{V_{i}\right\}\right)$. By comparing the cluster value $v_{c}\left(\mathcal{C}_{k}\right)$ with $v_{c}\left(\mathcal{C}_{k} \cup\left\{V^{*}\right\}\right)$, if $v_{c}\left(\mathcal{C}_{k} \bigcup\left\{V^{*}\right\}\right)>v_{c}\left(\mathcal{C}_{k}\right)$, add $V^{*}$ into $\mathcal{C}_{k}$, i.e., $\mathcal{C}_{k}=\mathcal{C}_{k} \bigcup\left\{V^{*}\right\}$, and set $\tau\left(V^{*}\right)=k$ and $\delta\left(V^{*}\right)=0$; otherwise, delete the first index in $\mathcal{L}\left(V^{*}\right)$ and update $\delta\left(V^{*}\right)$ and all the virtual clusters. Note that, whenever the individual information of a vertex in the interference-aware graph changes, the virtual clusters should correspondingly be updated. This iterative RB assignment process cycles until all the virtual clusters are empty, i.e., $\mathcal{C}_{k}^{\star}=\Phi$, with $k \in \mathcal{K}$. Finally, the RB assignment solution is composed of $\left\{\tau\left(V_{i}\right) \mid V_{i} \in \mathcal{V}\right\}$. The RSU announces the RB assignment solution to the corresponding communication links; then, the V2I and V2V communication links can perform their individual data transmission on the allocated RBs.

\section{Complexity Analysis}

According to Algorithm 1, as shown in Table I, the proposed interference-aware graph-based resource-sharing algorithm is processed in an iterative manner. Different initial states of the interference-aware graph constructed based on the current information of the communication links in the vehicular network will lead to a different number of iterations to obtain the final $\mathrm{RB}$ assignment solution and, thus, different computational complexity of the proposed algorithm. Thus, here, we simply focus on the worst case complexity of the proposed interferenceaware graph-based resource-sharing algorithm, which can sufficiently verify the efficiency of the proposed algorithm in reducing the complexity compared with the optimal resourcesharing scheme formulated in (5) and (6) with an exhaustive searching algorithm to obtain the optimal RB assignment solution. Based on the procedure of the proposed interferenceaware graph-based resource-sharing algorithm, the worst-case complexity can easily be calculated when considering the case that all the communication links experience all the virtual clusters and is given as

$$
\mathcal{C}_{\text {aware }}=\mathcal{O}\left(\frac{(M+N+1)(M+N) K}{2}\right)
$$

where we treat the computational complexity of the problem to find a maximum or minimum value from a cluster with $N$ values as $\mathcal{O}(N)$. Compared with the complexity of the optimal resource-sharing scheme calculated in (7), the proposed interference-aware graph-based resource-sharing algorithm can effectively reduce the computational complexity to obtain the $\mathrm{RB}$ assignment solution.

\section{INTERFERENCE-CLASSIFIED GRAPH-BASED RESOURCE-SHARING SCHEME}

Based on the simulation results in Section $\mathrm{V}$, we can see that the proposed interference-aware graph-based resource-sharing scheme can effectively assign the RBs to the V2I and V2V communication links and achieve a near-optimal performance in terms of the sum rate compared with the optimal solution formulated in (5) and (6). However, the construction of the interference-aware graph demands all the channel information of the communication and interference links, leading to a significant communication overhead. Therefore, in this section, we propose an interference-classified graph-based resourcesharing scheme in which the interference graph is constructed without the need of accurate interference channel information; instead, we use the classified interference based on the relative geographic location of different communication links. As illustrated in Fig. 3, the relative geographic location of two communication links can be divided into eight cases, mainly according to whether the receiver of one communication link is located within the interference range of the transmitter of the other communication link. In Fig. 3, parameters $R_{r}$ and $R_{v}$ represent the interference radius of the RSU and the vehicle, respectively. Note that, compared with the interference channel information, the relative geographic location of two communication links can easily be determined only by the current locations of the vehicles and the interference radii $R_{r}$ and $R_{v}$, which are predefined, leading to a much lower communication overhead.

\section{A. Interference-Classified Graph Construction}

Similar to the interference-aware graph, in the interferenceclassified graph, denoted by $\mathcal{G}_{i c}=\left(\mathcal{V}, \mathcal{E}_{i c}\right)$, each vertex in the vertices set $\mathcal{V}$ represents a V2I or V2V communication link, and each edge, which connects two vertices, in the edge set $\mathcal{E}_{i c}$ has a weight that characterizes the potential mutual interference between the two vertices. Each vertex, which is denoted by $V_{i}$, with $i=1,2, \ldots, M+N$, has four individual attributes, i.e., the link attribute, the resource attribute, the cluster attribute, and the neighbor attribute, where the link attribute, the resource attribute, and the cluster attribute are defined similar to those in the interference-aware graph. The neighbor attribute of vertex $V_{i}$, which is denoted by $\mathcal{V}_{i}^{\text {neighbor }}$, is a set of the vertices that are within the interference range of $V_{i}$.

Different from the edge weight in the interference-aware graph that can accurately be calculated by the collected interference channel-state information, in the interference-classified graph, we just classify the edge weight, which is denoted by $W_{V_{i}, V_{j}}$, with $V_{i}, V_{j} \in \mathcal{V}$, and $V_{i} \neq V_{j}$, into four levels, i.e., no interference, medium interference, significant interference, and infinity interference. The four interference levels between two vertices in the proposed interference-classified graph are classified based on the relative geographic location and the identity information of the considered two communication links and can be defined as follows.

- No interference. Neither receiver of the two communication links is located within the interference range of the transmitter of the other communication link, i.e., the cases shown in Fig. 3(a) and (e).

- Medium interference. Only one receiver of the two communication links is located within the interference range of the transmitter of the other communication link, i.e., the cases shown in Fig. 3(b), (c), (f), and (g). 


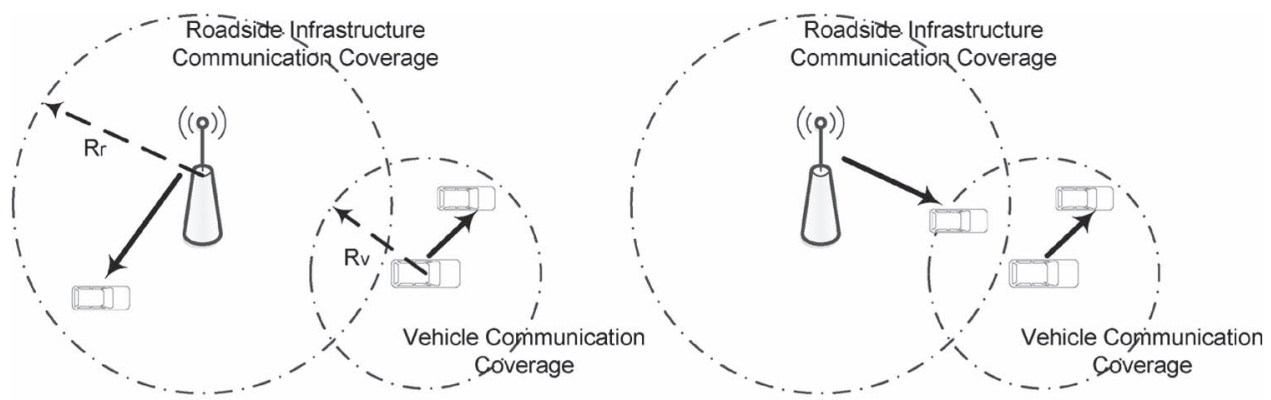

(a)

(b)

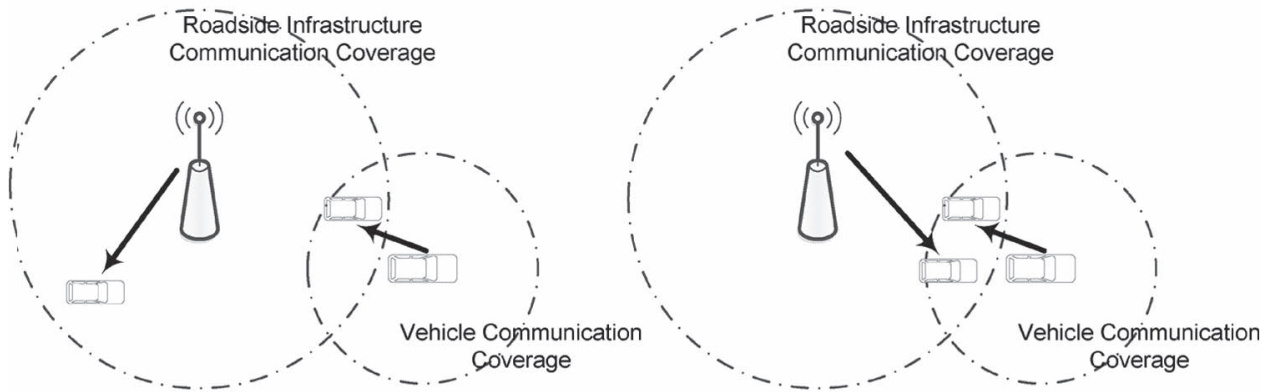

(c)

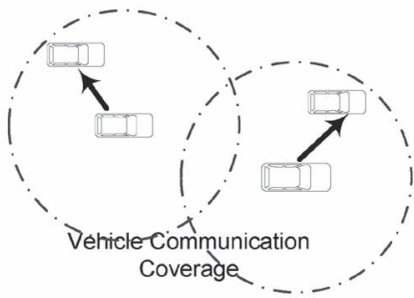

(e)

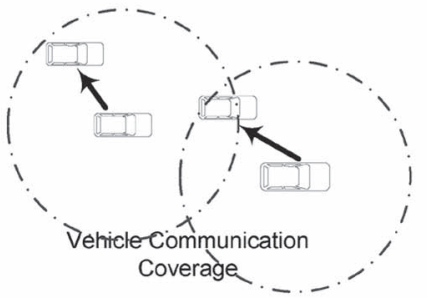

(g) (d)

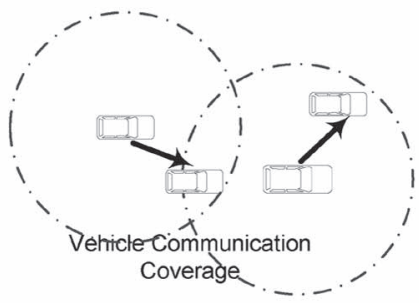

(f)

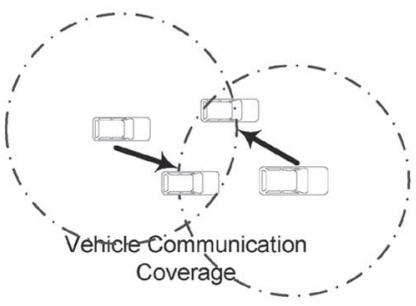

(h)

Fig. 3. Different cases for the relative geographic location of two different communication links.

- Significant interference. Both the receivers of the two communication links are located within the interference range of the transmitter of the other communication link, and at least one of the two communication links is a V2V communication link, i.e., the cases shown in Fig. 3(d) and (h).

- Infinity interference. If both the considered communication links are V2I links, the interference between them should be set to infinity or equal to a sufficiently large value to guarantee our assumption that resource sharing among different V2I communication links are forbidden.

The aforementioned analysis is performed for every pair of the vertices in the graph to determine the interference level of each corresponding edge. There are only four possible weight values of each edge in the proposed interference-classified graph, i.e., $W_{0}, W_{1}, W_{2}$, and $W_{n}$, which correspond to no interference, medium interference, significant interference, and infinity interference, respectively. In addition, the four weight values are ranked as $W_{0} \ll W_{1}<W_{2} \ll W_{n}$, which indicates different degrees of the interference. Therefore, the edge weight $W_{V_{i}, V_{j}}$ with $V_{i}, V_{j} \in \mathcal{V}$ and $V_{i} \neq V_{j}$ can be given as

$$
W_{V_{i}, V_{j}}= \begin{cases}W_{0}, & \text { If } V_{i} \notin \mathcal{V}_{j}^{\text {neighbor }}, V_{j} \notin \mathcal{V}_{i}^{\text {neighbor }} \\ W_{1}, & \text { If } V_{i} \notin \mathcal{V}_{j}^{\text {neighbor }}, V_{j} \in \mathcal{V}_{i}^{\text {neighbor }} \\ & \text { or } V_{i} \in \mathcal{V}_{j}^{\text {neighbor }}, V_{j} \notin \mathcal{V}_{i}^{\text {neighbor }} \\ W_{2}, & \text { If } V_{i} \in \mathcal{V}_{j}^{\text {neighbor }}, V_{j} \in \mathcal{V}_{i}^{\text {neighbor }} \\ & \text { and } V_{i} \in \mathcal{B} \text { or } V_{j} \in \mathcal{B} \\ W_{n}, & \text { If } V_{i} \in \mathcal{A} \text { and } V_{j} \in \mathcal{A} .\end{cases}
$$




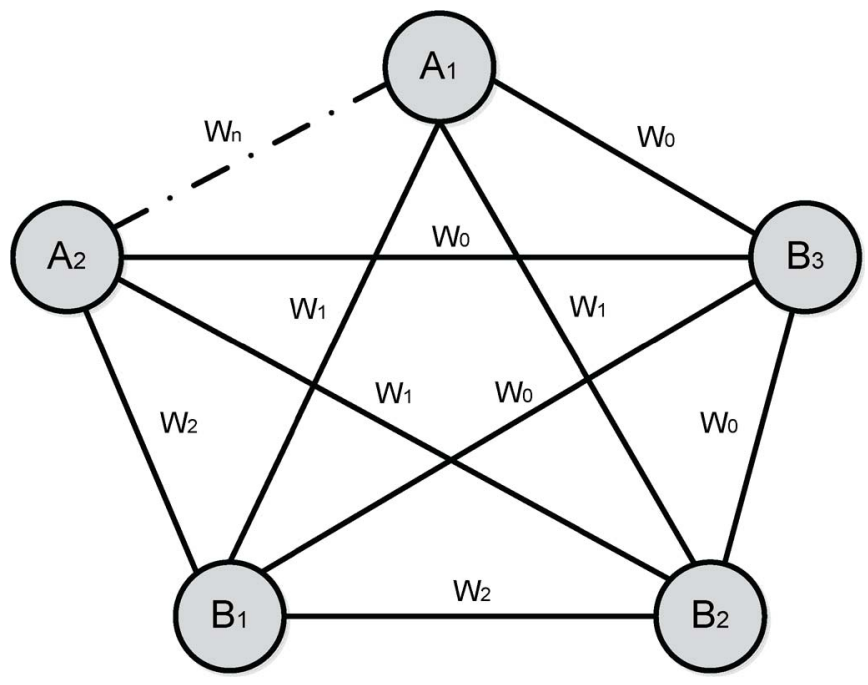

Fig. 4. Illustrative example of an interference-classified graph.

Then, the example of the interference-aware graph shown in Fig. 2 can be transformed into an interference-classified graph as shown in Fig. 4 based on the scenario in Fig. 1.

\section{B. Interference-Classified Graph-Based Resource-Sharing Algorithm}

Based on the constructed interference-classified graph, we propose an interference-classified graph-based resourcesharing algorithm. We also employ the concept of the interference value and the virtual cluster, which were defined in Section III-B, in this interference-classified graph-based resource-sharing algorithm. Note that the mutual interference in the interference-classified graph, i.e., the edge weight, cannot distinguish different channel responses on different RBs. Therefore, we assume that the mutual interference between two vertices $V_{i}$ and $V_{j}$ on $\mathrm{RB}_{k}$, which is denoted by $W_{V_{i}, V_{j}}^{k}$, is an equal division of the corresponding edge weight, i.e., $W_{V_{i}, V_{j}}^{k}=W_{V_{i}, V_{j}} / K$, with $V_{i}, V_{j} \in \mathcal{V}, V_{i} \neq V_{j}$, and $k \in \mathcal{K}$. Therefore, the interference value in the interference-classified graph is given as

$$
\begin{aligned}
v_{i}\left(\mathcal{C}_{k}\right) & =\sum_{V_{i}, V_{j} \in \mathcal{C}_{k}, V_{i} \neq V_{j}} W_{V_{i}, V_{j}}^{k} \\
& =\sum_{V_{i}, V_{j} \in \mathcal{C}_{k}, V_{i} \neq V_{j}} \frac{W_{V_{i}, V_{j}}}{K} .
\end{aligned}
$$

As described in Algorithm 2, as shown in Table II, the procedure of the interference-classified graph-based resource-sharing algorithm is similar to the interference-aware graph-based resource-sharing algorithm. After the construction and initialization of the interference-classified graph, the iterative resource assignment process starts. Considering the cluster $\mathcal{C}_{k}$ of the $\mathrm{RB}_{k}$, we first select a vertex $V^{*}$ from the virtual cluster $\mathcal{C}_{k}^{\star}$ to satisfy $V^{*}=\arg \min _{V_{i} \in \mathcal{C}_{k}^{\star}} v_{i}\left(\mathcal{C}_{k} \bigcup\left\{V_{i}\right\}\right)$. Note that there is no cluster value concept in this algorithm due to the lack of the
TABLE II

ALGORITHM 2: INTERFERENCE-CLASSIFIED GRAPH-BASED RESOURCE-SHARING ALGORITHM

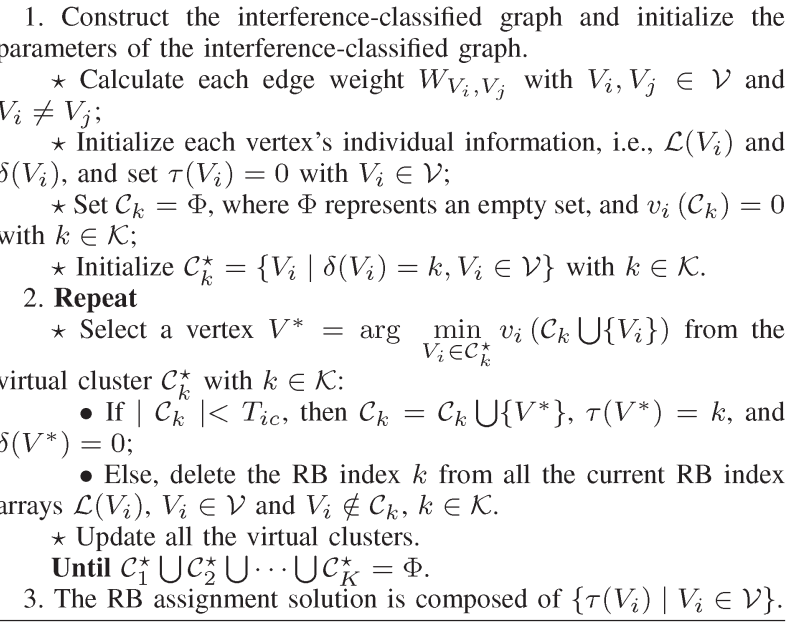

accurate interference channel information. Therefore, we limit the number of vertices in one cluster by a threshold $T_{i c}$ to avoid too much vertices joining in one cluster, which may degrade the system performance due to the increasing interference when the number of a vertices in a cluster grows up. If $\left|\mathcal{C}_{k}\right|<T_{i c}$, where $\left|\mathcal{C}_{k}\right|$ represents the current number of vertices in $\mathcal{C}_{k}$, add $V^{*}$ into $\mathcal{C}_{k}$, i.e., $\mathcal{C}_{k}=\mathcal{C}_{k} \bigcup\left\{V^{*}\right\}$, and set $\tau\left(V^{*}\right)=k$ and $\delta\left(V^{*}\right)=0$. Note that the RB index $k$ will be deleted from all the current RB index vectors $\mathcal{L}\left(V_{i}\right)$ with $V_{i} \in \mathcal{V}, V_{i} \notin \mathcal{C}_{k}$, and $k \in \mathcal{K}$ once $\left|\mathcal{C}_{k}\right|=T_{i c}$. Whenever the individual information of a vertex in the interference-classified graph changes, the virtual clusters should correspondingly be updated. In addition, this iterative RB assignment process cycles until all the virtual clusters are empty, i.e., $\mathcal{C}_{k}^{\star}=\Phi$, with $k \in \mathcal{K}$. Finally, the RB assignment solution is composed of $\left\{\tau\left(V_{i}\right) \mid V_{i} \in \mathcal{V}\right\}$. The RSU announces the $\mathrm{RB}$ assignment solution to the corresponding communication links; then, the V2I and V2V communication links can perform their individual data transmission on the allocated RBs.

\section{Complexity Analysis}

According to Algorithm 2, shown in Table II, the worst case complexity of the proposed interference-classified graph-based resource-sharing algorithm can also be calculated when considering the case that all the communication links experience all the virtual clusters and equal to that of the interferenceclassified graph-based resource-sharing algorithm, i.e.,

$$
\mathcal{C}_{\text {classified }}=\mathcal{O}\left(\frac{(M+N+1)(M+N) K}{2}\right) .
$$

Note that, although the interference-classified graph-based resource-sharing algorithm and the interference-aware graphbased resource-sharing algorithm have the same worst case complexity, the communication overhead of the interferenceclassified graph-based resource-sharing algorithm is much lower than that of the interference-aware graph-based 
TABLE III

Simulation PARAMETERS

\begin{tabular}{|l|l|}
\hline Parameters & Value \\
\hline Channel Bandwidth $W$ & $20 \mathrm{MHz}$ \\
Total RB number $K$ & 10 \\
RSU's Transmit Power $p_{i}$ & $20 \mathrm{dBm}$ \\
Vehicle's Transmit Power $p_{v}$ & $5 \mathrm{dBm}$ \\
RSU's Interference Radius $R_{r}$ & $100 \mathrm{~m}$ \\
Vehicle's Interference Radius $R_{v}$ & $10 \mathrm{~m}$ \\
Path Loss Factor & 3 \\
Small Scale Fading & Rayleigh fading coefficient \\
Noise Power Spectral Density & $-174 \mathrm{dBm} / \mathrm{Hz}$ \\
Noise Figure & $5 \mathrm{~dB}$ \\
The Threshold $T_{i c}$ & 3 \\
Percentage of V2I Communication Links & $30 \%$ \\
Velocity of the Vehicles & $0 \mathrm{~km} / \mathrm{h}-100 \mathrm{~km} / \mathrm{h}$ \\
Time Varying Channel Model & Autoregressive Model in [25] \\
Information Feedback Delay $\tau_{\text {Delay }}$ & $1 \mathrm{~ms}$ \\
\hline
\end{tabular}

resource-sharing algorithm, because the interference-classified graph can be constructed without the detailed interference channel-state information among different communication links, which saves a lot of communication overhead. Thus, the interference-classified graph-based resource-sharing algorithm is more suitable to be implemented in practical vehicular networks due to its low computational complexity and low communication overhead.

\section{Simulation Results and Analysis}

To evaluate the utility of the developed underlaying resourcesharing communication mode and the proposed interference graph-based resource-sharing schemes, we conduct the following simulations. Consider a $20 \mathrm{~m} \times 500 \mathrm{~m}$ rectangle area that denotes the road, where the RSU is located at the center of one of the long edges, and the vehicles are randomly distributed within the area, with a random velocity of $0-100 \mathrm{~km} / \mathrm{h}$. The detailed simulation parameters are presented in Table III.

Fig. 5 compares the network sum rate of the two proposed interference graph-based resource-sharing schemes, the optimal resource-sharing scheme that is an exhausted searching scheme based on the optimization resource-sharing problem formulated in (5) and (6), and the traditional greedy resource assignment scheme that assigns the RBs to the communication links with the highest transmission channel gain on the corresponding RBs. Note that, in the traditional greedy resource assignment scheme, each RB can be allocated to only one communication link to guarantee the orthogonality of different data transmission links in the investigated vehicular network. In fact, this greedy resource assignment scheme can be formulated based on [9]. We generalize the optimal subcarrier allocation policy for V2I communications in [9] to obtain the traditional greedy resource assignment scheme by equivalently treating V2I and V2V communication links. Based on Fig. 5, it is clear that the proposed interference-aware graph-based resource-sharing scheme can achieve an approaching performance compared with that of the optimal resource-sharing scheme but has much lower computational complexity to obtain an effective RB

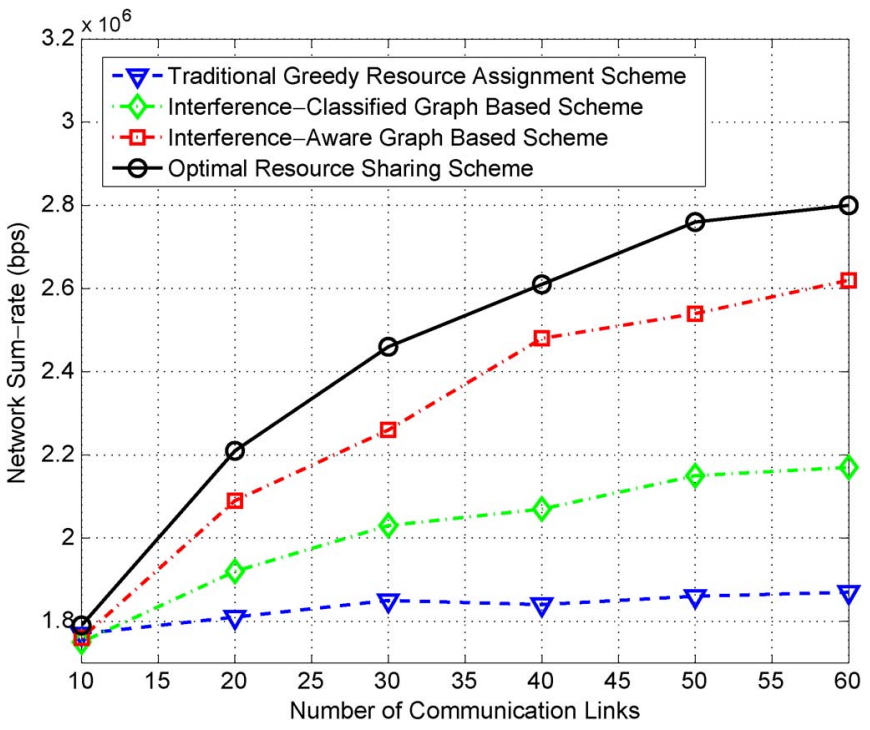

Fig. 5. Sum-rate performance with different resource management schemes.

assignment solution. In addition, compared with the traditional greedy resource assignment scheme, in which resource sharing by different communication links is forbidden, all the three resource-sharing schemes have a significant performance gain in terms of the sum rate, particularly when the traffic load is heavy. This result verifies the utility of the proposed underlaying resource-sharing communication mode for vehicular networks.

However, based on Fig. 5, we should also note that, when the number of the communication links is 10 , i.e., equal to the number of the RBs, the traditional greedy resource assignment scheme achieves higher performance compared with the interference-classified graph-based resource-sharing scheme. This is because, in such a case, the traditional greedy resource assignment scheme can always fully utilize all the RBs based on the exact channel-state information of the communication links, whereas the interference-classified graph-based resourcesharing scheme assigns the RBs to the communication links based on the classified interference level between different communication links, which is not the exact channel-state information of the interference links that may lead to a loss in performance. This indicates that the advantage of the underlaying resource-sharing communication mode does not effectively appear when the number of the communication links is small. However, for the optimal resource-sharing scheme, due to the optimization resource-sharing problem formulated in (5) and (6), the obtained sum rate will always be better than or at least equal to that of the traditional greedy resource assignment scheme.

As shown in Fig. 6, we compare the following two proposed interference graph-based resource-sharing schemes, i.e., the interference-aware graph-based resource-sharing scheme and the interference-classified graph-based resource-sharing scheme, as well as the optimal resource-sharing scheme in terms of the cumulative distribution function (cdf) of the SINR at a heavy traffic load situation where the number of communication links is 50. Based on Fig. 6, it is obvious that the interference-aware graph-based resource-sharing scheme 


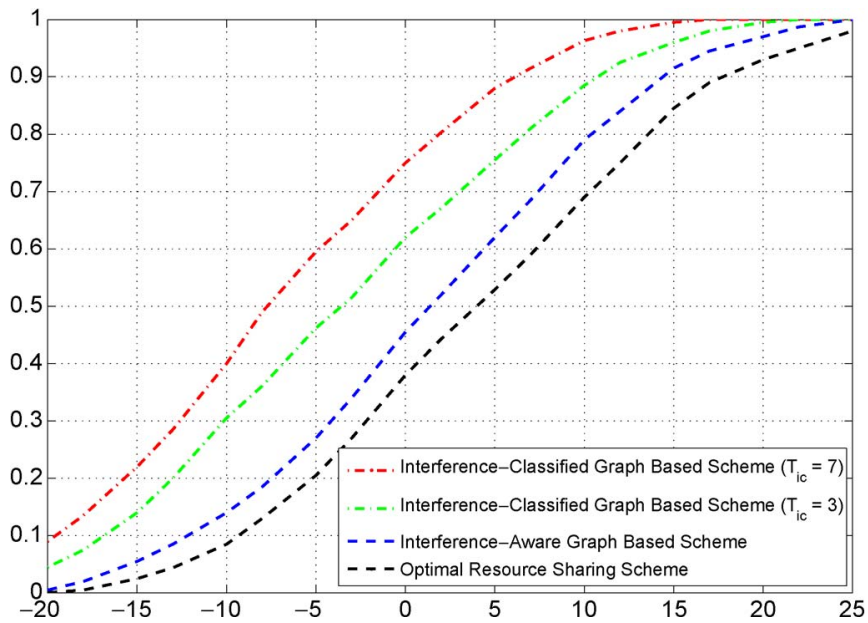

Fig. 6. CDF of the SINR for the case of 50 communication links.

achieves better performance than the interference-classified graph-based resource-sharing scheme due to the accurate interference information of the interference-aware graph. However, the interference-aware graph-based resource-sharing scheme has much higher communication overhead than the interference-classified graph-based resource-sharing scheme. Therefore, the interference-classified graph-based resourcesharing scheme is easier to implement into practical vehicular networks. In addition, Fig. 6 illustrates the impact of the factor $T_{i c}$ on the performance of the interference-classified graphbased resource-sharing scheme. In Fig. 6, we can see that the factor $T_{i c}$ will significantly influence the performance of the interference-classified graph-based resource-sharing scheme. Fig. 6 also clearly shows that a larger value of $T_{i c}$ will degrade the performance of the interference-classified graphbased resource-sharing scheme. This is because the larger the value of $T_{i c}$ is, the higher the interference each RB's cluster has.

\section{CONCLusion}

This paper has investigated the resource-sharing problem in vehicular networks, where both V2V and V2I communication links coexist. A novel underlaying resource-sharing communication mode for vehicular networks has been proposed. Moreover, the corresponding resource-sharing problem has been solved with low computational complexity by using the graph theory, and two interference graph-based resource-sharing schemes have been developed. The proposed interferenceclassified graph-based resource-sharing scheme can significantly reduce the communication overhead in the proposed interference-aware graph-based resource-sharing scheme and, thus, is more suitable for practical applications. Simulation results have demonstrated that the proposed interference-aware graph-based resource-sharing scheme can achieve near-optimal performance in terms of the network sum rate, whereas the proposed interference-classified graph-based resource-sharing scheme can achieve suboptimal performance but still expresses better network sum rate than the traditional orthogonal communication mode in vehicular networks.

\section{REFERENCES}

[1] S. Olariu and M. C. Weigle, Vehicular Networks: From Theory to Practice. London, U.K.: Chapman \& Hall, 2009, ser. Comput. and Inf. Sci.

[2] Y. Toor, P. Muhlethaler, and A. Laouiti, "Vehicle ad hoc networks: Applications and related technical issues," IEEE Commun. Surveys Tuts., vol. 10, no. 3, pp. 74-88, Third Quar., 2008.

[3] C.-X. Wang, X. Cheng, and D. I. Laurenson, "Vehicle-to-vehicle channel modeling and measurements: Recent advances and future challenges," IEEE Commun. Mag., vol. 47, no. 11, pp. 96-103, Nov. 2009.

[4] X. Cheng, C.-X. Wang, H. Wang, X. Gao, X.-H. You, D. Yuan, B. Ai, Q. Huo, L. Song, and B. Jiao, "Cooperative MIMO channel modeling and multilink spatial correlation properties," IEEE J. Sel. Areas Commun., vol. 30, no. 2, pp. 388-396, Feb. 2012.

[5] C.-X. Wang, X. Hong, X. Ge, X. Cheng, G. Zhang, and J. S. Thompson, "Cooperative MIMO channel models: A survey," IEEE Commun. Mag., vol. 48, no. 2, pp. 80-87, Feb. 2010.

[6] X. Cheng, C.-X. Wang, D. I. Laurenson, S. Salous, and A. V. Vasilakos, "An adaptive geometry-based stochastic model for nonisotropic MIMO mobile-to-mobile channels," IEEE Trans. Wireless Commun., vol. 8, no. 9, pp. 4824-4835, Sep. 2009.

[7] Z. Zhang, G. Mao, and B. Anderson, "On the information propagation process in mobile vehicular ad hoc networks," IEEE Trans. Veh. Technol., vol. 60, no. 5, pp. 2314-2325, Jun. 2011.

[8] J. A. Juan, V.-A. Javier, and G.-H. Joan, "Control-based scheduling with QoS support for vehicle to infrastructure communications," IEEE Wireless Commun., vol. 16, no. 6, pp. 32-39, Dec. 2009.

[9] H. Zhang, Y. Ma, D. Yuan, and H.-H. Chen, "Quality-of-service driven power and subcarrier allocation policy for vehicular communication networks," IEEE J. Sel. Areas Commun., vol. 29, no. 1, pp. 197-206, Jan. 2011

[10] B. Bai, W. Chen, K. B. Letaief, and Z. Cao, "Low-complexity outageoptimal distributed channel allocation for vehicle-to-vehicle communications," IEEE J. Sel. Areas Commun., vol. 29, no. 1, pp. 161-172, Jan. 2011.

[11] Q. Wang, P. Fan, and K. B. Letaief, "On the joint V2I and V2V scheduling for cooperative VANETs with network coding," IEEE Trans. Veh. Technol., vol. 61, no. 1, pp. 62-73, Jan. 2012.

[12] S. C. Ng, W. Zhang, Y. Zhang, Y. Yang, and G. Mao, "Analysis of access and connectivity probabilities in vehicular relay networks," IEEE J. Sel. Areas Commun., vol. 29, no. 1, pp. 140-150, Jan. 2011.

[13] J. Miller, "Vehicle-to-vehicle-to-infrastructure (V2V2I) intelligent transportation system architecture," in Proc. IEEE Intell. Veh. Symp., Jun. 2008, pp. 715-720.

[14] J. Miller, "Analysis of vehicle lane changes for determining fastest paths in the V2V2I ITS architecture," in Proc. 11th Int. IEEE ITSC, Dec. 2008, pp. 1207-1212.

[15] S.-Y. Pyun, D.-H. Cho, and J.-W. Son, "Downlink resource allocation scheme for smart-antenna-based V2V2I communication system," in Proc. IEEE VTC-Fall, Sep. 2011, pp. 1-6.

[16] K. Doppler, M. Rinne, C. Wijting, C. B. Ribeiro, and K. Hugl, "Device-todevice communication as an underlay to LTE-Advanced networks," IEEE Commun. Mag., vol. 47, no. 12, pp. 42-49, Dec. 2009.

[17] C.-H. Yu, K. Doppler, C. B. Ribeiro, and O. Tirkkonen, "Resource-sharing optimization for device-to-device communication underlaying cellular networks," IEEE Trans. Wireless Commun., vol. 10, no. 8, pp. 2752-2763, Aug. 2011.

[18] M. Haenggi, J. G. Andrews, F. Baccelli, O. Dousse, and M. Franceschetti, "Stochastic geometry and random graphs for the analysis and design of wireless networks," IEEE J. Sel. Areas Commun., vol. 27, no. 7, pp. 10291046, Sep. 2009.

[19] Y.-J. Choi, J. Kim, and S. Bahk, "QoS-aware selective feedback and optimal channel allocation in multiple shared channel environments," IEEE Trans. Wireless Commun., vol. 5, no. 11, pp. 3278-3286, Nov. 2006.

[20] R. Y. Chang, Z. Tao, J. Zhang, and C.-C. J. Kuo, "Multicell OFDMA downlink resource allocation using a graphic framework," IEEE Trans. Veh. Technol., vol. 58, no. 7, pp. 3494-3507, Sep. 2009.

[21] T. Qiu, W. Xu, Z. He, K. Niu, and B. Tian, "Graph-based spectrum sharing for multiuser OFDM cognitive radio networks," in Proc. Int. Conf. WCSP, Nov. 2011, pp. 1-5.

[22] A. Jalali, R. Padovani, and R. Pankaj, "Data throughput of CDMA-HDR: A high-efficiency high-data-rate personal communication wireless system," in Proc. IEEE VTC-Spring, Tokyo, Japan, 2000, pp. 1854-1858.

[23] J.-G. Choi and S. Bahk, "Cell-throughput analysis of the proportional fair scheduler in the single-cell environment," IEEE Trans. Veh. Technol., vol. 56, no. 2, pp. 766-778, Mar. 2007. 
[24] D. A. Plaisted, "Some polynomial and integer divisibility problems are NP-hard," in Proc. 17th Annu. Symp. Found. Comput. Sci., 1976, pp. 264-267.

[25] K. E. Baddour and N. C. Beaulieu, "Autoregressive modeling for fading channel simulation," IEEE Trans. Wireless Commun., vol. 4, no. 4, pp. 1650-1662, Jul. 2005.

[26] C. F. Mecklenbräuker, A. F. Molisch, J. Karedal, F. Tufvesson, A. Paier, L. Bernadó, T. Zemen, O. Klemp, and N. Czink, "Vehicular channel characterization and its implications for wireless system design and performance," Proc. IEEE, vol. 99, no. 7, pp. 1189-1212, Jul. 2011.

[27] A. Gamst and K. Ralf, "Computational complexity of some interference graph calculations," IEEE Trans. Veh. Technol., vol. 39, no. 2, pp. 140149, May 1990

[28] L. Song and J. Shen, Evolved Cellular Network Planning and Optimization for UMTS and LTE. Boca Raton, FL, USA: CRC, 2010.

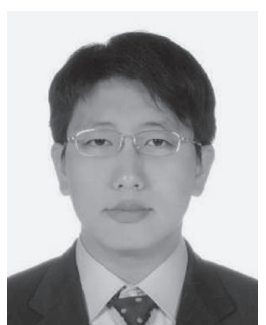

Rongqing Zhang (S'11) received the B.S. degree in electronic engineering in 2009 from Peking University, Beijing, China, where he is currently working toward the Ph.D. degree, with a major in wireless communications and signal processing.

He has published two book chapters and more than 20 papers in refereed journals and conference proceedings. His research interests include physical-layer security, device-to-device communications, game theory, graph theory, and distributed resource allocation.

Mr. Zhang received the Academic Scholarship for Doctoral Candidates from the Ministry of Education of China in 2012 and the Best Paper Award at the 2012 IEEE International Conference on Intelligent Transportation Systems Telecommunications.

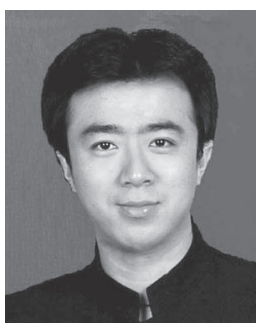

Xiang Cheng (S'05-M'10) received the Ph.D. degree in 2009 from Heriot-Watt University and the University of Edinburgh, Edinburgh, U.K.

In 2010, he was a Lecturer with Peking University, Beijing, China, where he has been an Associate Professor since 2012. His research interests include mobile propagation channel modeling and simulation, next-generation mobile cellular systems, intelligent transportation systems, and hardware prototype development and practical experiments. He has published more than 60 research papers in journals and conference proceedings.

Dr. Cheng received the Best Paper Award at the 2012 IEEE International Conference on Intelligent Transportation Systems Telecommunications, the 2009 Chinese National Award for Outstanding Oversea Ph.D. Students for his academic excellence and outstanding performance, and the Postgraduate Research Thesis Prize from Heriot-Watt University and the University of Edinburgh in 2009. He served as a Symposium Cochair and a member of Technical Program Committee for several international conferences.

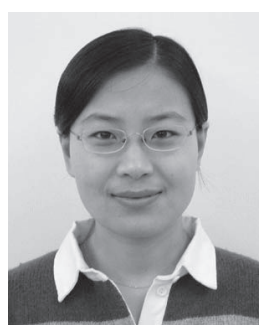

Qi Yao received the B.Sc. and M.Eng. degrees in communication and information systems from Shandong University, Shandong, China, in 1997 and 2000, respectively.

From 2000 to 2001, she was a Research Assistant with the Technical University of Hamburg-Harburg, Hamburg, Germany. From 2001 to 2006, she was a Research Fellow with the University of Agder, Grimstad, Norway. Since 2007, she has been with Heriot-Watt University, Edinburgh, U.K., where she is currently a Research Associate. Her research interests include wireless communications, wireless channel modeling and simulation, and multiple-input-multiple-output systems. She has published more than 40 papers in refereed journals and conference proceedings.

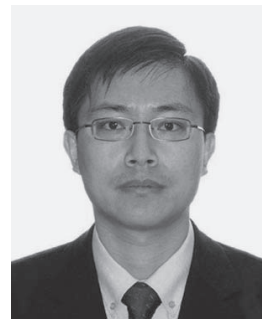

Cheng-Xiang Wang (S'01-M'05-SM'08) received the B.Sc. and M.Eng. degrees in communication and information systems from Shandong University, Shandong, China, in 1997 and 2000, respectively, and the $\mathrm{Ph} . \mathrm{D}$. degree in wireless communications from Aalborg University, Aalborg, Denmark, in 2004.

Since 2005, he has been with Heriot-Watt University, Edinburgh, U.K., first as a Lecturer, then as a Reader in 2009, and as a Professor in 2011. He is also an Honorary Fellow of the University of Edinburgh and a Chair/Guest Professor with Shandong University; Huazhong University of Science and Technology, Wuhan, Hubei, China; and Southeast University, Nanjing, China. From 2001 to 2005, he was a Research Fellow with the University of Agder, Grimstad, Norway. In 2004, he was a Visiting Researcher with Siemens AG-Mobile Phones, Munich, Germany. From 2000 to 2001, he was a Research Assistant with the Technical University of Hamburg-Harburg, Hamburg, Germany. He is the Editor of one book. He has published one book chapter and over 170 papers in refereed journals and conference proceedings. His research interests include wireless channel modeling and simulation, green communications, cognitive radio networks, vehicular communication networks, large multiple-input-multiple-output (MIMO), cooperative MIMO, and Beyond-Fourth-Generation wireless communications.

Prof. Wang is a Fellow of the Institution of Engineering and Technology and the Higher Education Academy and a member of the Engineering and Physical Research Council Peer Review College. He has served as an editor for eight international journals, including the IEEE TRANSACTIONS ON VEHICULAR TECHNOLOGY (since 2011) and the IEEE TRANSACTIONS ON WIRELESS COMMUNiCATIONS (2007-2009). He was the lead Guest Editor for the IEEE Journal on Selected AREAS in Communications Special Issue on Vehicular Communications and Networks. He has served as a Technical Program Committee (TPC) Member, TPC Chair, and General Chair for more than 70 international conferences. He received the Best Paper Awards at the 2010 IEEE Global Telecommunications Conference, the 13th IEEE International Conference on Communication Technology in 2011, and the 2012 International Conference on Intelligent Transport Systems Telecommunications.

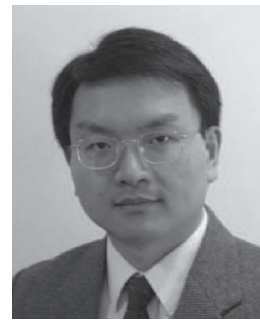

Yang Yang (S'99-M'02-SM'10) received the B.Eng. and M.Eng. degrees from Southeast University, Nanjing, China, in 1996 and 1999, respectively, and the Ph.D. degree from the Chinese University of Hong Kong, Shatin, Hong Kong, in 2002.

$\mathrm{He}$ is a member of the Founding Team for the School of Information Science and Technology, ShanghaiTech University, Shanghai, China, which was jointly established by the Shanghai Municipal Government and the Chinese Academy of Sciences (CAS), Beijing, China. He is currently a Professor with the CAS Shanghai Institute of Microsystem and Information Technology and serves as the Director with the Shanghai Research Center for Wireless Communications. Prior to joining ShanghaiTech University, he was a Senior Lecturer with the Department of Electronic and Electrical Engineering, University College London, London, U.K.; a Lecturer with the Department of Electronic and Computer Engineering, Brunel University, Middlesex, U.K.; and an Assistant Professor with the Department of Information Engineering, Chinese University of Hong Kong, Shatin, Hong Kong. His research interests include wireless ad hoc and sensor networks, wireless mesh networks, nextgeneration mobile cellular systems, intelligent transport systems, and wireless testbed development and practical experiments.

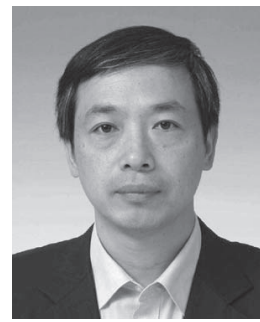

Bingli Jiao (M'05) received the B.S. and M.S. degrees from Peking University, Beijing, China, in 1983 and 1988, respectively, and the Ph.D. degree from the University of Sarrbruecken, Sarrbruecken, Germany, in 1995.

In 1995, he was an Associate Professor with Peking University, where he has been a Professor since 2000. His interests include communication theory and techniques and sensor design. 